\title{
Research on Security Level Evaluation Method for Cascading Trips Based on WSN
}

\author{
Hui-Qiong Deng, ${ }^{1,2}$ Jie Luo $\mathbb{D}^{1,2}{ }^{1,2}$ Ku-Chi Chang, ${ }^{3,4}$ Qin-Bin Li, ${ }^{1}$ Rong-Jin Zheng, ${ }^{1}$ \\ and Pei-Qiang $\mathrm{Li}^{1}$ \\ ${ }^{1}$ School of Electronic Electrical and Physics, Fujian University of Technology, Fuzhou 350118, China \\ ${ }^{2}$ Fujian Provincial University Engineering Research Center for Simulation Analysis and Integrated Control of Smart Grid, \\ Fujian, China \\ ${ }^{3}$ College of Mechanical \& Electrical Engineering, National Taipei University of Technology, Taipei 10608, Taiwan \\ ${ }^{4}$ Department of Business Administration, North Borneo University College, Sabah 88400, Malaysia
}

Correspondence should be addressed to Jie Luo; 550461238@qq.com

Received 28 December 2020; Revised 8 March 2021; Accepted 26 April 2021; Published 19 May 2021

Academic Editor: Pei-Wei Tsai

Copyright (c) 2021 Hui-Qiong Deng et al. This is an open access article distributed under the Creative Commons Attribution License, which permits unrestricted use, distribution, and reproduction in any medium, provided the original work is properly cited.

\begin{abstract}
In recent years, the application of wireless sensor networks (WSN) in power systems has received a great deal of attention. As we all know, the most important issue for the power system is security and stability, especially due to the massive outages caused by cascading trips. Therefore, in today's era, from the perspective of cascading trips, how to effectively use WSN to analyze and evaluate the security level of the power grid is an important direction for future power development. In this paper, an algorithm based on the WSN collection of online data to calculate the corresponding security level of the system is proposed for the cascading trip phenomenon, to achieve the online evaluation of the cascading trips. First, this paper proposes a hybrid layered network structure based on WSN for monitoring system and details the acquisition of power grid parameters by its acquisition layer. Secondly, combined with the manifestation of cascading trips and the action equation of current-type line backup protection, the mathematical representation of the grid cascading trips is given, and the mathematical form corresponding to the critical situation is strictly proved, and an index for evaluating the security level of the power grid is proposed and then further combined with the actual physical constraints of the power grid and the establishment of a mathematical model for calculating the security level of the grid cascading trips. For this model, this paper relies on evolution particle swarm optimization (EPSO) to give specific ideas for solving the model. Finally, a case analysis is performed by the IEEE39 node system and the results of the case show the effectiveness of the model and method.
\end{abstract}

\section{Introduction}

With the development of microsensor technology, microelectronics technology, wireless communication technology, and computer technology, wireless sensor networks (WSN) with the functions of information collection, processing, and transmission emerge as the times require [1]. Currently, WSN technology is also gradually penetrating into the power industry, and its application prospects have attracted much attention.

As a system that provides clean secondary energy to cities and villages, today's power system has established a very strong connection to society as a whole. Therefore, the safe and reliable operation of the power system becomes an essential topic, and to ensure the safe and reliable operation of the power system, first of all, we need to quickly and accurately collect the power system operation data. Then, analyze on this basis to determine whether the settings of the various operating parameters of the power system are reasonable, whether they need to be adjusted, and how to adjust them. Conventional power systems typically use potential transformers and current transformers to collect power data, gather them to the monitoring center of the substation, and then transmit the data to a remote dispatch center with 
corresponding communication facilities. The advantages of this method are high security, stability, the anti-interference ability of data transmission, small size, and lightweight. However, this method also has some obvious disadvantages, such as the existence of certain limitations, comprehensive wiring difficulties, long construction period, high cost, limited monitoring range, poor scalability, equipment maintenance difficulties, and a series of other problems [2].

WSN can efficiently and quickly collect and transmit the main data of the whole system of the power system due to its low cost, adaptability to the environment, efficient collection of information, and full coverage of the monitoring area [3]. WSN has emerged as the ideal choice to meet the new challenges of power grid parameter monitoring technology. The wireless sensor installed on the power equipment is used to complete electrical information acquisition and preprocessing., and the synchronized data collected will be transmitted to the monitoring center through the wireless communication network, which analyzes and processes the information [4]. WSN for power grid parameters to provide more flexible and complete monitoring solutions can deal with the power grid development and application of special requirements and can realize centralized management of multitype power grid parameter monitoring [5]. The above features of WSN for the overall perspective of the power system security analysis and defense are extremely advantageous, especially for the analysis and prevention of cascading trips.

Generally speaking, the cascading trips of the power system is an event caused by the interaction between components, which in severe cases can lead to vicious blackouts, so cascading trips have attracted much attention. At present, researchers have done a great deal of work in the field of defense and control regarding cascading trips. The literature [6] addresses the problem of cascading trips, by predicting the state of the monitoring node set in the process of cascading trips; traction control is implemented for nodes in abnormal states to inhibit the propagation of failure in the network. However, it is difficult to apply it in practice due to insufficient consideration of the operating characteristics of the grid. The literature [7] proposes a wide-area collaborative precontrol method based on the theory of multi-intelligent systems and the analysis of offline cross-sectional power transmission limits. However, the influence of the current operation state on the outage probability of power system components is ignored. The literature [8] developed a model of cascading trip network interaction with information network edges as initial faults and proposed a vector construction method for false data attacks based on parameter estimation. Finally, corresponding defense measures are proposed based on false data attacks. However, the dynamic characteristics of the power grid are not considered. The literature [9] proposes a cascading trip path search and warning model based on the characteristics of the power grid information physical fusion system. However, there is the problem of inaccurate system data.

Given the actual characteristics of WSN and power grid cascading trips, this paper proposes a method to measure nodal power injection using WSN and analyze the security of the power grid for the expected initial failure to trigger cas- cading failure. The second and third parts of this paper mainly introduce the application of WSN in power system and the monitoring system based on WSN; the fourth part gives the mathematical equation to judge the cascading trips of the power grid; the fifth part mainly introduces how to use the node injection power data collected by WSN to analyze the security level of the power grid for cascading failure, and gives the specific analysis model; the sixth part mainly gives the solution algorithm and process for the given model; the seventh part gives an example based on the IEEE39 node system to verify and analyze the method in this paper.

\section{Combination of WSN and Power Grid Monitoring}

WSN is the core of the Internet of Things technology. It is one of the most cutting-edge technologies to realize the acquisition and transmission of various signals through low-power self-organizing and adaptive wireless sensor nodes [10]. The traditional wired communication wiring is cumbersome, the line is easy to aging, and the cost is high, while the wireless sensor network fully meets the speed requirements of power equipment condition monitoring, perfectly solves these shortcomings, reduces the cost of power operation and maintenance costs, and improves the stability and efficiency of power system operation. It promotes the application and rapid development of wireless sensor network technology in the power grid and lays a solid foundation for the efficient and rapid construction of power system network framework in the future and the improvement of user satisfaction.

.In the power system, the use of WSN technology to establish a remote monitoring system, at any time to monitor the status of power equipment data, to help operators on-line security assessment of the status of power equipment, abnormal response to the characteristics of the quantity, to take the necessary measures to avoid the occurrence of serious failure. In the distribution network relay protection, the wireless current sensor using WSN technology not only solves the problem of possible subcurrent saturation of the current transformer but also is easy to install, while the current data of the line is accurately and quickly collected by WSN to avoid the initial fault triggering the current overload protection and causing the relay device to operate incorrectly [11]. In summary, the combination of WSN and grid monitoring is a new trend in the future development of smart power grids.

\section{Monitoring System Based on WSN}

At present, the power system monitoring network based on WSN is mainly linear distribution, which usually arranges sensor nodes on transmission lines and relay nodes on towers and sends the collected information through the sensor nodes of the lines to the relay nodes of the towers, which are processed by the relay nodes and forwarded to the substations. This monitoring network has disadvantages such as poor effectiveness and can cause uneven load distribution. The use of a layered network architecture avoids the 
problems of high cost, low reliability, poor scalability, and limited transmission rate brought about by the existing monitoring systems that rely entirely on mobile communication networks. Therefore, in the development of smart power grids, the introduction of the WSN, combined with fiber optic Ethernet, can make full use of existing resources and protect existing investment, which is a more suitable choice in the current situation.

3.1. Structure of the Monitoring Network. This paper proposes a hybrid layered network structure to monitor the power system network by using the methods of literature $[12,13]$, combining the wired (optical fiber) and wireless (ZigBee and cellular) technology. The structure of the network is shown in Figure 1.

The whole network structure is divided into the acquisition layer, convergence layer, and teleportation layer. The acquisition layer is composed of a large number of sensor nodes, which is responsible for collecting electrical information on the transmission line, and a relay node with strong processing ability is arranged on each tower. The convergence layer consists of these relay nodes that are responsible for receiving and processing ordinary node data on the pole and tower. The teleportation layer is composed of representative nodes, substations, and monitoring centers, with wireless ZigBee connections between relay nodes and between relay nodes and substations, and data transmission from substations and representative nodes to monitoring centers via optical fiber and cellular networks, respectively. This hierarchical structure has the characteristics of strong network extensibility, strong effectiveness, and easy centralized management, which can meet the application requirements of the emerging smart power grids.

3.2. Acquisition of the Power Grid Parameters. The power grid parameters, which serve as accurate indexes for evaluation and feedback on the operational status of the power grid, must be dynamically monitored in real time. In this paper, with the help of new sensor technology, fast, accurate, and comprehensive realization of wireless acquisition of power grid parameters. Therefore, this paper focuses on the acquisition layer of the monitoring network structure. The hardware system design of the acquisition layer is shown in Figure 2.

As shown in Figure 2, in the acquisition layer, the wireless sensor acquisition nodes deployed in the power grid are responsible for voltage and current acquisition and processing and wireless transmission. It mainly includes a data acquisition module, a wireless transceiver circuit, and a power supply circuit. The power grid parameter acquisition node collects current signals and voltage signals through high-precision current transformers and voltage transformers and then processes them through analog conditioning circuits and inputs them to the sampling unit of CC2530 to complete the A/D conversion of the signals. Finally, wireless communication is realized by an RF transceiver. The power module is powered by two dry batteries.

The voltage phasor of the node and the current phasor of all the branches connected to it are obtained by converting the measured sample values into frequency domain signals:

$$
\left\{\begin{array}{l}
\dot{\mathbf{U}}=\frac{2}{N} \sum_{k=0}^{N-1} u_{k} e^{-j 2 \pi / N k}=\mathbf{U}_{\mathbf{r}}+j \mathbf{U}_{\mathrm{d}}, \\
\dot{\mathbf{I}}=\frac{2}{N} \sum_{k=0}^{N-1} i_{k} e^{-j 2 \pi / N k}=\mathbf{I}_{\mathrm{r}}+j \mathbf{I}_{\mathrm{d}},
\end{array}\right.
$$

where $u_{k}$ and $i_{k}$ denote the $k$ th voltage sampling value and current sampling value, respectively; $N$ is the total number of samples; $\mathbf{U}_{\mathrm{r}}$ and $\mathbf{I}_{\mathrm{r}}$ denote the real part of the phasor; and $\mathbf{U}_{\mathrm{d}}$ and $\mathbf{I}_{\mathrm{d}}$ denote the imaginary part of the phasor.

Based on the voltage phasor and current phasor obtained above, the nodal injection power can be further calculated using these data, and its calculation formula is as follows:

$$
\left\{\begin{array}{l}
S_{i j}=\dot{U}_{i} \times\left(\dot{I}_{i j}\right)^{*}, \\
S_{i}=\sum_{j \in \mathrm{i}} S_{i j},
\end{array}\right.
$$

where $U_{i}$ is the voltage phasor of node $i ; \dot{I}_{i j}$ is the current flowing through branch road $L_{i j} ; S_{i j}$ is the flow power on the $i$-side of the branch road $L_{i j}$; and $S_{i}$ is the injection power of node $i$.

\section{Basic Idea for Evaluating Power Grid Security Level Based on WSN}

As mentioned above, the WSN is used to get the power injection data of the power grid nodes, and then, the data is transmitted to the monitoring center through a hierarchical structure. This chapter will introduce how to use the nodal injection power data to help the monitoring personnel to evaluate the safety level of the cascading trips, and give the relevant expression and mathematical model.

4.1. Mathematical Representation of Power Grid for Cascading Trips. The cascading trips of the power grid is usually after the initial failure branch road is removed; due to the redistribution of power flow, the backup protection action in the branch road except the initial failure branch road is caused.The cascading trips of the power grid is usually caused by the action of backup protection in branch roads other than the initial failure branch road due to the redistribution of power flow after the removal of the initial failure branch road. Taking the current protection as an example, if the branch road $L_{a}$ in a power grid has an initial failure at a certain time, then whether any branch road $L_{b}$ in the remaining system in the power grid will have a cascading trip after the branch road $L_{a}$ is cut off; it can be judged by whether the current detected by its configured backup protection enters the action zone of the protection. The branch road $L_{b}$ is between node $i$ and node $j$. If the node $i$-side of the branch road $L_{b}$ is equipped with current-type backup protection, the equation shown 


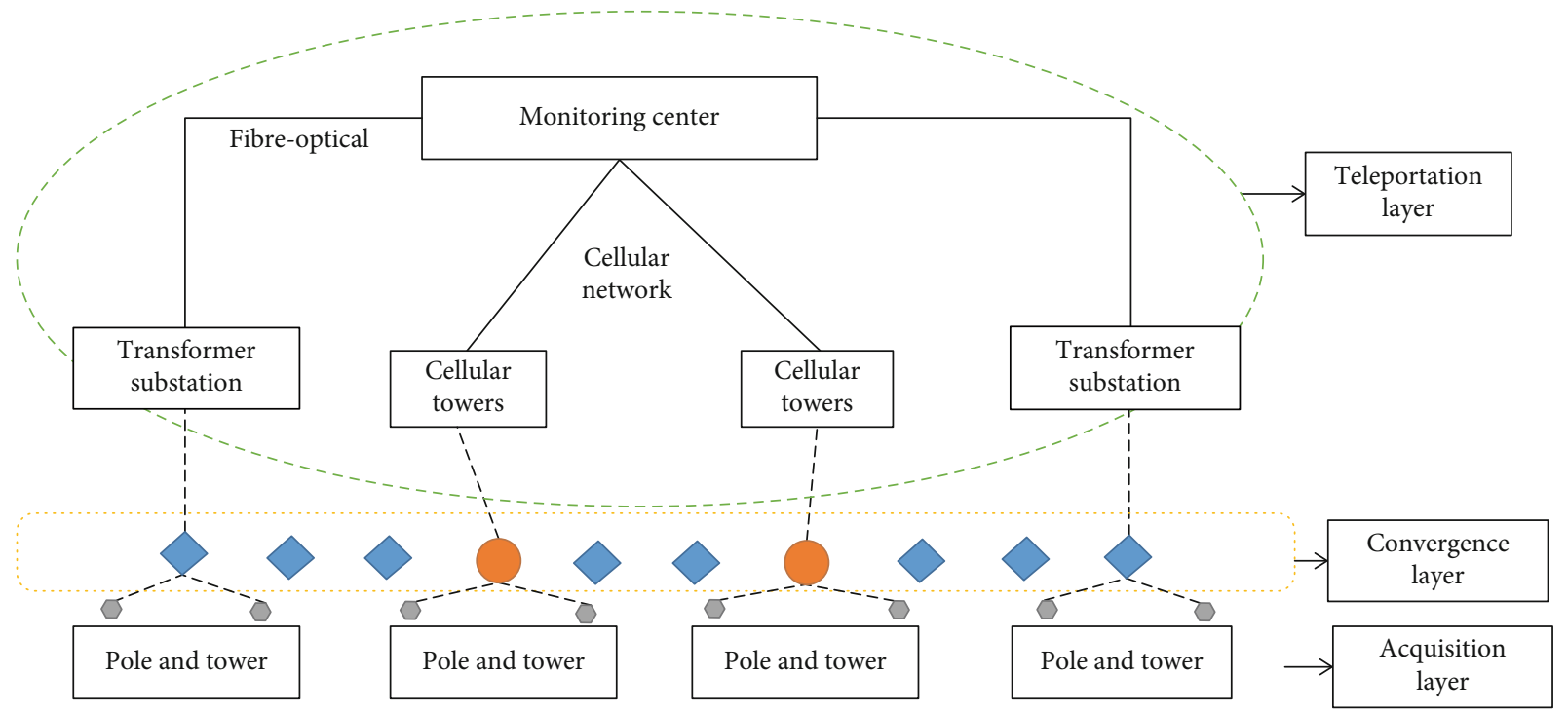

Ordinary node

Relay node

Representative node

FIGURE 1: Hybrid hierarchical network structure.

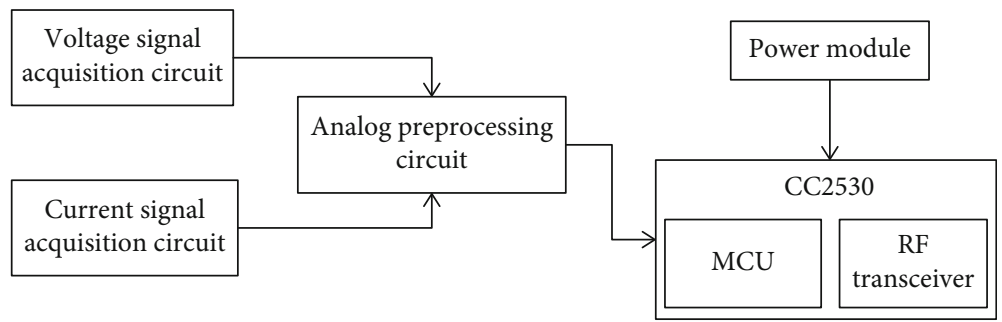

FIgURE 2: Hardware diagram of the acquisition layer.

in Equation (3) can be defined according to the protection setting value and the measured current.

$$
I_{b \cdot \text { dist }}^{i}=I_{b \cdot s e t}^{i}-I_{b \cdot m}^{i}
$$

Here, $I_{b \cdot m}^{i}$ is the current measured by the backup protection on the $i$-side of the branch road $L_{b}, I_{b \text { set }}^{i}$ is the setting value of the backup protection on the $i$-side of the branch road $L_{b}$, and $I_{b \text {.dist }}^{i}$ is the distance between $I_{b \cdot s e t}^{i}$ and $I_{b \cdot m}^{i}$.

From Equation (3) and the action characteristics of the protection, it can be seen that when $I_{b \text {.dist }}^{i}>0$, the $i$-side of the $L_{b}$ will not have cascading trips, and when $I_{b \text {.dist }}^{i}<0$, the $i$-side of the branch road $L_{b}$ will have cascading trips, and when $I_{b \text {-dist }}^{i}=0$, the $i$-side of the branch $\operatorname{road} L_{b}$ is at the boundary of cascading trips.

Similarly, if the $j$-side of branch road $L_{b}$ is equipped with current-type backup protection, the cascading trips of branch road $L_{b}$ will be caused after the protection action. The form of a similar equation (3) can be used to judge it, and the super- script $i$ in Equation (3) can be replaced by $j$. For branch road $L_{b}$, either the $i$-side or $j$-side shows cascading trips; then, the branch will have cascading trips.

According to the judgment equation of branch road cascading trips given in Equation (3), all branch roads except the initial fault branch road in the power grid are considered, and then, the judgment of cascading trips at the power grid stratification plane can be further given. In fact, from the power grid stratification plane, according to the performance of the power grid cascading trips, the cascading trip of at least one branch road can be regarded as the cascading trips of the power grid. At this time, the state of the power grid can be called the state of the power grid cascading trips. If at least one branch road of the power grid is at the boundary of the cascading trips and the remaining branch roads are in the safe state except for those branch roads in the boundary state of the cascading trips, the state of the power grid can be called the critical state of the power grid cascading trips. Further, in addition to the state in which cascading trips occur in the power grid and the critical state, the state in which the power grid is in which cascading trips do not occur is the safe state. 
To give a further mathematical description of the above state, the various types of backup protection on the remaining branch roads of the power grid except the initial faulted branch roads can be considered uniformly, and the variable $C$ is used to represent $I$ in Equation (3) uniformly, and all the backup protections are numbered uniformly. For any branch road $L_{b}$, the specific numbering can be done according to the following rules: if the $i$-side of the branch road $L_{b}$ is equipped with current-type backup protection, it will be recorded as $C_{b \text {.dist }}^{(1)}$, and if the $j$-side of the branch road $L_{\mathrm{b}}$ is also equipped with current-type backup protection, it will

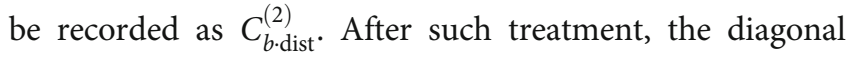
matrix of the following form can be given:

$$
\mathbf{C}=\operatorname{diag}\left(C_{1 \cdot \text { dist }}, \cdots, C_{b \cdot \text { dist }}^{(1)}, C_{b \cdot \text { dist }}^{(2)}, \cdots, C_{m \cdot \text { dist }}^{(2)}\right),
$$

where $m$ is the total number of branches in the power grid except for the initial fault branch roads. Equation (4) is written according to the current-type backup protection on both sides of each branch. If a branch road is only equipped with backup protection on one side, the corresponding elements can be removed from Equation (4).

After Equation (4) is given, according to the above analysis, when at least one branch road in the power grid meets $C_{b \text { dist }}^{(k)}<0$, the power grid is in the state of cascading trips. Here, $k$ can be taken as 1 or 2 according to the situation. When Equation (5) is satisfied, the power grid is in a safe state.

$$
C_{b . \text { dist }}^{(k)}>0, \quad k=1,2, b=1,2, \cdots, m .
$$

Further, when Equation (6) is satisfied, the power grid is just in the critical state of cascading trips.

$$
\left\{\begin{array}{l}
|\mathbf{C}|=0, \\
C_{b \cdot \text { dist }}^{(k)}>0, \quad k=1,2, b=1,2, \cdots, m,
\end{array}\right.
$$

where $|\mathbf{C}|$ is the determinant value of matrix $\mathbf{C}$.

For the critical state described by Equation (6), this paper gives the following proof: let the set of all operating states of the power grid be represented by $T$, and let $T_{1}$ be the set of operations without any branch road cascading trips, $T_{2}$ be the set of the power grid in the critical state of cascading trips, and $T_{3}$ be the set where cascading trips occur but does not belong to the critical state. According to this division, it is easy to know that $T=T_{1} \cup T_{2} \cup T_{3}$, and $T_{1} \cap T_{2}=\varnothing, T_{1} \cap$ $T_{3}=\varnothing$, and $T_{2} \cap T_{3}=\varnothing$. Let $\Omega 1$ be an operation state satisfying Equation (6); if $\Omega 1 \in T_{1}$, then all branch roads in the power grid must satisfy Equation (5), and $|\mathbf{C}|$ is not zero, which is contradictory to Equation (6), so there must be $\Omega 1 \in T_{1}$; if $\Omega 1 \in T_{3}$, then at least one branch road $L_{b}$ satisfies $C_{b \text {.dist }}^{(k)}<0$ according to the definition of $T_{3}$, which is also contradictory to Equation (6), so there must be $\Omega 1 \in T_{2}$.

4.2. Mathematical Model of the Security Level of the Power Grid for Cascading Trips. Among the various states of the power grid for cascading trips, the critical state is a key state. Obviously, in the actual operation, to ensure the safety level of the power grid for cascading trips, the power grid should be as far away from the critical state as possible. Through the analysis of the power flow equation of the power grid, it can be seen that the redistribution of power flow is mainly determined by the nodal injection power before the initial fault. Therefore, to keep the power grid away from the critical state, it is necessary to keep the nodal injection power away from the nodal injection power corresponding to the critical state.

Among all such nodal injection power combinations, the one closest to the current operating state of the power grid is a special combination. If the nodal injection power combination in the current operating state of the power grid is far away from this combination, then the nodal injection power in the current operating state of the power grid must be farther away from the remaining nodal injection power combinations that make the grid in the critical state of cascading trips.

If the combination of nodal injection power in the current operating state of the grid is represented by the vector $\mathbf{S}^{\prime}$ and the combination of nodal injection power that makes the grid in the critical state of the cascading trips is represented by the vector $\mathbf{S}$, then the distance between the two can be represented by the following equation:

$$
d(\mathbf{S})=\left\|\mathbf{S}^{\prime}-\mathbf{S}\right\|
$$

Here, $\left\|\mathbf{S}^{\prime}-\mathbf{S}\right\|$ denotes the parametric number for $\mathbf{S}^{\prime}-\mathbf{S}$.

Based on the above analysis, the vector $\mathbf{S}$ obtained by taking the minimum value of $d(\mathbf{S})$ is used as the combination of the nodal injection power that, makes the power grid in the critical state of cascading trips, and for the convenience of analysis, the minimum value of $d(\mathbf{S})$ is here denoted as $f(\mathbf{S}$ ), as shown in the following equation:

$$
f(\mathbf{S})=\min d(\mathbf{S})=\min \left\|\mathbf{S}^{\prime}-\mathbf{S}\right\|,
$$

Here, $f(\mathbf{S})>0$, The higher the $f(\mathbf{S})$, the safer the power grid, so $f(\mathbf{S})$ reflects the level of security of the power grid for cascading trips; $f(\mathbf{S})$ can be represented as a security index. The mentioned method is security level prediction for cascading trips based on nodal injection power, which later in this paper will be called the SLP method.

From the process before and after the occurrence of cascading trips in the power grid, when the power injected into the power grid $\mathbf{S}$ meets Equation (8), it also needs to meet certain physical constraints, including various equation constraints and inequality constraints. The equation constraint condition shall include the power flow constraint relationship of the power grid before and after the initial failure, which can be abbreviated to the form shown in equation (9):

$$
\left\{\begin{array}{l}
h^{0}(\mathbf{x})=0 \\
h^{a}(\mathbf{x})=0 .
\end{array}\right.
$$


Here, $\mathbf{x}$ is the state variable of the system. $h^{0}$ is the mapped relationship of the power flow constraint satisfied by the grid before the initial failure. $h^{a}$ is the mapped relationship of the power flow constraint satisfied by the grid after the initial failure branch road $L_{a}$ is removed.

For the relevant inequality constraints, these include generator output constraints, node voltage constraints, and line power constraints before and after the initial failure. They will be written in an abbreviated form as

$$
m^{0}(\mathbf{x}) \leq 0
$$

Summing up the previous analysis, you get the equation shown in the following equation:

$$
\left\{\begin{array}{l}
f(\mathbf{S})=\min \left\|\mathbf{S}^{\prime}-\mathbf{S}\right\| \\
h^{0}(\mathbf{x})=0 \\
h^{a}(\mathbf{x})=0 \\
m^{0}(\mathbf{x}) \leq 0 \\
|\mathbf{C}|=0 \\
C_{b . \text { dist }}^{(k)} \geq 0, \quad k=1,2 b=1,2, \cdots, m
\end{array}\right.
$$

The model provided in Equation (11) is an optimization model that describes the problem of how to find the closest state to the current operating state among the running states that can trigger cascading trips of the power grid, with the state represented by the nodal injection power. Obviously, the nodal injection power corresponding to the closest critical state to the current operating state of the power grid can be obtained using Equation (11), as well as the power grid's current operating state for cascading trip security level index.

4.3. Evaluation Idea for Power Grid Security Level. The existing various optimization algorithms have been widely applying in several fields, such as brain storm optimization algorithm (BSO) [14], memetic algorithm (MA) [15, 16], firefly algorithm (FA) $[17,18]$, and particle swarm optimization algorithm (PSO) [19]. Considering that the PSO is easy to implement programmatically, straightforward, and quick and suitable for solving complex optimization problems that are difficult to be solved by various classical optimization algorithms, this paper draws on the evolution particle swarm optimization (EPSO) of literature [20] to solve the model, whose iterative formula is shown below.

$$
\left\{\begin{array}{l}
\mathbf{v}_{i}^{k+1}=w \mathbf{v}_{i}^{k}+b 1 r 1\left(\mathbf{P}_{\text {best.i }}-\mathbf{y}_{i}^{k}\right)+b 2 r 2\left(\mathbf{g}_{\text {best }}-\mathbf{y}_{i}^{k}\right), \\
\mathbf{y}_{i}^{k+1}=\mathbf{y}_{i}^{k}+\mathbf{v}_{i}^{k+1} .
\end{array}\right.
$$

Here, $\mathbf{y}_{i}{ }^{k}$ is the iterative position of particle $i$ at the $k$ th time; $\mathbf{v}_{i}{ }^{k}$ is the iterative velocity of particle $i$ at the $k$ th time; $\mathbf{P}_{\text {best } i}$ is the individual optimal position of particle $i$; $\mathbf{g}_{\text {best }}$ is the population optimal position; $w$ is the inertia coefficient, which decreases linearly from 0.9 to $0.1 ; b_{1}$ and $b_{2}$ are accel- eration constants, which are all set to 2 ; and $r_{1}$ and $r_{2}$ are $[0,1]$ randomly distributed random numbers.

To cooperate with EPSO, all the equation constraints in Equation (12) are combined and written in the form $e(\mathbf{x})=$ 0 , and all the inequality constraints in Equation (12) are combined and written in the form $k(\mathbf{x}) \geq 0$. Then, the following objective function with the penalty factor may be further given [21]. The constrained problem is transformed into an unconstrained problem by adding a penalty function. In this way, the solution is faster, and the operation is simple.

$$
f^{\prime}(\mathbf{S})=f(\mathbf{S})+\sum_{i} \frac{1}{\alpha_{i}}\left[\min \left(0,-e_{i}(\mathbf{x})\right)\right]^{2}+\sum_{j} \frac{1}{\beta_{j}}\left[\min \left(0,-k_{j}(\mathbf{x})\right)\right]^{2} .
$$

Here, $e_{i}(\mathbf{x})$ is the component $i$ in $e(\mathbf{x})$, and $k_{j}(\mathbf{x})$ is the component $j$ in $k(\mathbf{x})$. $\alpha$ and $\beta$ are penalty factors, and the value of the penalty factor depends on the actual situation.

In this way, Equation (13) can be used to obtain the nodal injection power in the critical state according to the EPSO, as well as the corresponding grid security level index, where the particle position $y$ corresponds to the nodal injection power vector S.txin Equation (13) can be understood as an intermediate variable that satisfies the system constraint

To sum up the above, this paper mainly follows the following ideas to assess the security level of the power grid for the cascading trips, and its flow of ideas is shown in Figure 3.

Further, as seen in the previous analysis that after the security index $f(\mathbf{S})$ is calculated, a circle can be obtained with the current state of the power grid as the center of the sphere and the radius of the security index $f(\mathbf{S})$. When the operating state of the power grid is inside this sphere, the power grid is secure. For example, in a 2-node system, the nodal injection power of the grid is assumed to be active power, as shown in Figure 4.

In Figure $4, \mathbf{P}_{a}$ is the nodal injection power combination of the current state of the grid, denoted by $\bullet$; the nodal injection power combination of the critical state is denoted by $*$, and $\mathbf{P}_{b}$ is the nodal injection power combination of the critical state closest to $\mathbf{P}_{a}$; with $\mathbf{P}_{a}$ as the center of the circle and the distance between $\mathbf{P}_{a}$ and $\mathbf{P}_{b}$ as the radius to build circle $D$, the region within circle $D$ is $C_{1}$, and the remaining region is $\mathrm{C}_{2}$.

As shown in Figure 4, when the current operating state of the power grid is in zone $C_{1}$ but deviates from $\mathbf{P}_{a}$ if the power grid is hit by the initial failure and cascading trips have not occurred, you can use WSN to make further verification. The specific idea is as follows: when the actual power grid is working in zone $C_{1}$ but deviates from $\mathbf{P}_{a}$ and the initial failure does occur, the use of WSN quickly collects the current of each branch of the power grid and compares it with the protection of the fixed value, to determine whether the grid has cascading trips; if there are no cascading trips, then the method of this paper is reliable to determine whether cascading trips occur in the power grid. If not, it indicates that the method in this paper is reliable.. 


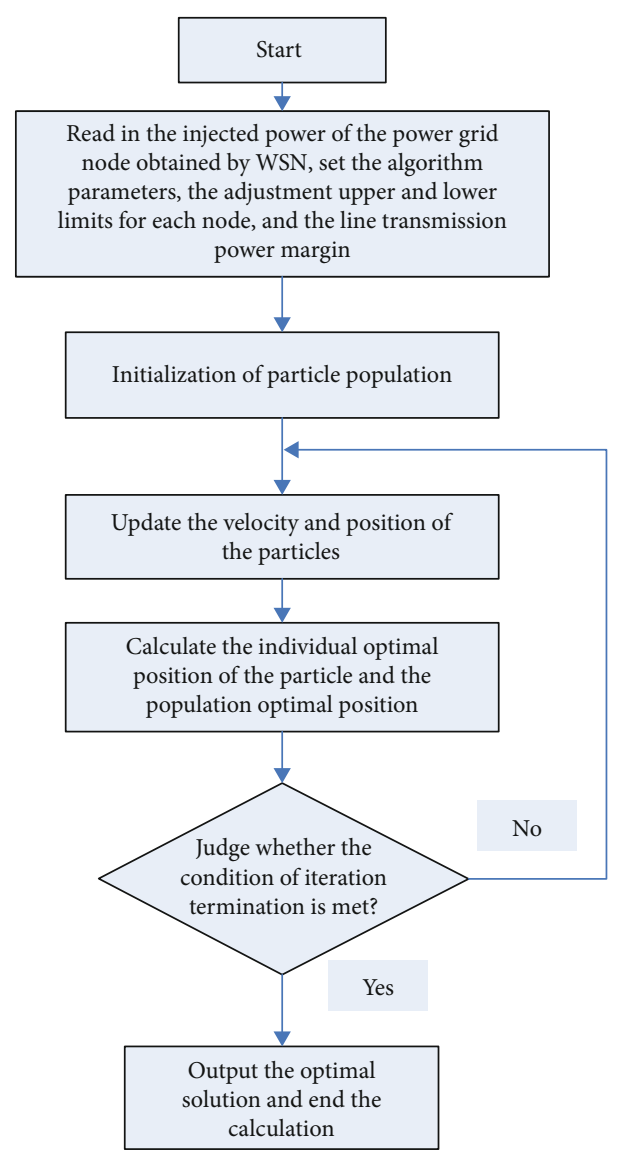

Figure 3: Algorithm flow.

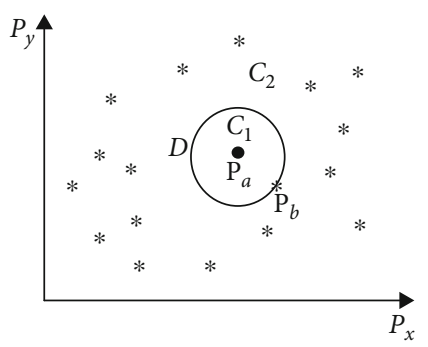

FIGURE 4: Security zone for the current operating state of the power grid.

\section{Example Analysis}

For illustration and validation of the previously proposed analytical model, this paper uses the IEEE39 node system for the algorithm demonstration; the wiring of the IEEE39 node system is shown in Figure 5. In the following example analysis, the results calculated in this paper are expressed as per-unit value, the reference capacity is considered as $100 \mathrm{MVA}$, and the reference voltage is consistent with the reference voltage of the IEEE36 bus data given in the literature [21].

According to the previous ideas in this paper, grid security assessment using WSN focuses on the analysis of the grid based on getting the nodal injection power, due to the former

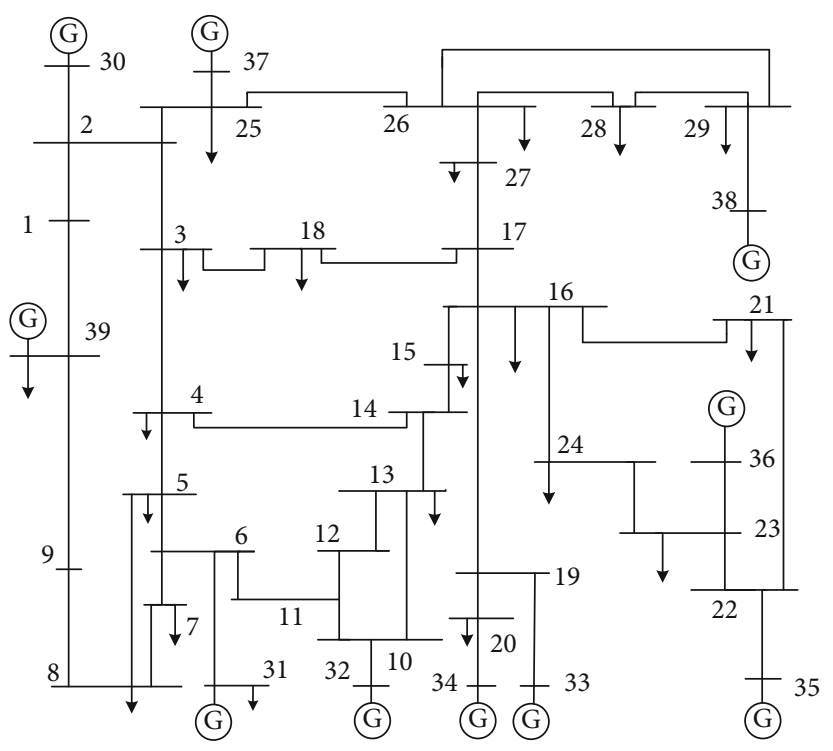

FIGURE 5: Diagram of IEEE39 node system.

advantages of WSN, which can obtain the nodal injection power of the entire power grid with a short delay and with high efficiency and accuracy. To focus on the verification of the evaluation method, this example assumes that the IEEE39 node system has configured WSN according to the structure shown in Figure 1, and the voltage phasor and current phasor collected through WSN are shown in Table 1 and Table 2, and their values are expressed as per-unit value. According to the obtained voltage and current, the nodal injection power is calculated according to Equation (2). Due to space limitations, the nodal injection power data will not be listed here. In the calculation, considering the actual characteristics of power flow, this paper mainly combines the PV node's active power, PQ node's active power, and reactive power to form the nodal injection power combination vector $\mathbf{S}$.

With the solution idea of EPSO, it performed under the analysis program compiled in the Matlab environment. Suppose that the initial failure branch road is the branch road between node 5 and node 8, namely, branch road $L_{5,8}$. Each branch road of the system shown in Figure 5 configures the current-type backup protection, and the constant value of protection is $5.77 \mathrm{KA}$.

For the values of $\alpha_{i}$ and $\beta_{j}$ in Equation (13), when they correspond to the constraint relationship in Equation (10), their values are taken as 0.01 ; when they correspond to the constraint relationship in Equation (6), $\alpha_{i}$ is taken as 0.0085 , and $\beta_{j}$ is taken as 0.0065 . In the latter case, the smaller value of $\alpha_{i}$ and $\beta_{j}$ is mainly because the constraint conditions corresponding to these two penalty factors are more closely related to the cascading trips. In the calculation, it is taken into account that $f(\mathbf{S})$ in Equation (13) is usually small and $|\mathbf{C}|$ is relatively large. For the convenience of analysis, the value of $f(\mathbf{S})$ is multiplied by $10 e 5$ in the procedure in this paper, while the value of $|\mathbf{C}|$ is divided by $10 e 5$, and the results below and the results of this paper will be given according to this requirement. 
TABLe 1: Voltage phasor collected by WSN.

\begin{tabular}{lccccc}
\hline $\begin{array}{l}\text { Node } \\
\text { number }\end{array}$ & $\begin{array}{c}\text { Voltage } \\
\text { phasor }\end{array}$ & $\begin{array}{c}\text { Node } \\
\text { number }\end{array}$ & $\begin{array}{c}\text { Voltage } \\
\text { phasor }\end{array}$ & $\begin{array}{c}\text { Node } \\
\text { number }\end{array}$ & $\begin{array}{c}\text { Voltage } \\
\text { phasor }\end{array}$ \\
\hline 1 & 1.047 & 14 & 1.011 & 27 & 1.037 \\
2 & 1.048 & 15 & 1.015 & 28 & 1.050 \\
3 & 1.030 & 16 & 1.032 & 29 & 1.049 \\
4 & 1.004 & 17 & 1.034 & 30 & 1.047 \\
5 & 1.005 & 18 & 1.031 & 31 & 0.982 \\
6 & 1.007 & 19 & 1.050 & 32 & 0.983 \\
7 & 0.997 & 20 & 0.991 & 33 & 0.997 \\
8 & 0.996 & 21 & 1.011 & 34 & 1.012 \\
9 & 1.028 & 29 & 1.049 & 35 & 1.049 \\
10 & 1.017 & 30 & 1.047 & 36 & 1.063 \\
11 & 1.012 & 31 & 0.982 & 37 & 1.027 \\
12 & 1 & 32 & 0.983 & 38 & 1.026 \\
13 & 1.014 & 33 & 0.997 & 39 & 1.030 \\
\hline
\end{tabular}

TABLE 2: Current phasor collected by WSN.

\begin{tabular}{|c|c|c|c|c|c|}
\hline $\begin{array}{l}\text { Branch } \\
\text { head node } \\
\text { number }\end{array}$ & $\begin{array}{l}\text { Branch } \\
\text { end node } \\
\text { number }\end{array}$ & $\begin{array}{l}\text { Current } \\
\text { phasor }\end{array}$ & $\begin{array}{l}\text { Branch } \\
\text { head node } \\
\text { number }\end{array}$ & $\begin{array}{l}\text { Branch } \\
\text { end node } \\
\text { number }\end{array}$ & $\begin{array}{c}\text { Current } \\
\text { phasor }\end{array}$ \\
\hline 1 & 2 & 1.152 & 16 & 24 & 0.899 \\
\hline 1 & 39 & 1.281 & 17 & 18 & 1.789 \\
\hline 2 & 3 & 3.667 & 17 & 27 & 0.202 \\
\hline 2 & 25 & 2.457 & 21 & 22 & 5.968 \\
\hline 3 & 4 & 1.388 & 22 & 23 & 0.644 \\
\hline 3 & 18 & 0.411 & 23 & 24 & 3.425 \\
\hline 4 & 5 & 1.642 & 25 & 26 & 0.753 \\
\hline 4 & 14 & 2.640 & 26 & 27 & 2.669 \\
\hline 5 & 6 & 4.775 & 26 & 28 & 1.349 \\
\hline 5 & 8 & 3.211 & 26 & 29 & 1.840 \\
\hline 6 & 7 & 4.311 & 28 & 29 & 3.382 \\
\hline 6 & 11 & 3.478 & 11 & 12 & 0.274 \\
\hline 7 & 8 & 1.895 & 13 & 12 & 0.322 \\
\hline 8 & 9 & 0.885 & 31 & 6 & 5.780 \\
\hline 9 & 39 & 0.164 & 32 & 10 & 6.709 \\
\hline 10 & 11 & 3.478 & 33 & 19 & 7.129 \\
\hline 10 & 13 & 2.955 & 34 & 20 & 5.128 \\
\hline 13 & 14 & 2.977 & 35 & 22 & 6.122 \\
\hline 14 & 15 & 0.373 & 36 & 23 & 5.311 \\
\hline 15 & 16 & 3.202 & 37 & 25 & 5.272 \\
\hline 16 & 17 & 2.031 & 30 & 2 & 2.365 \\
\hline 16 & 19 & 4.368 & 38 & 29 & 8.041 \\
\hline 16 & 21 & 3.196 & 20 & 19 & 4.373 \\
\hline
\end{tabular}

Figure 6 shows the comparison of the minimum $f$ calculated by EPSO and PSO according to the same parameter setting. The total number of iterations is 200 , and the horizontal ordinate in the figure indicates the number of iterations, while the vertical ordinate is the minimum $f^{\prime}(\mathbf{S})$ obtained for each iteration.

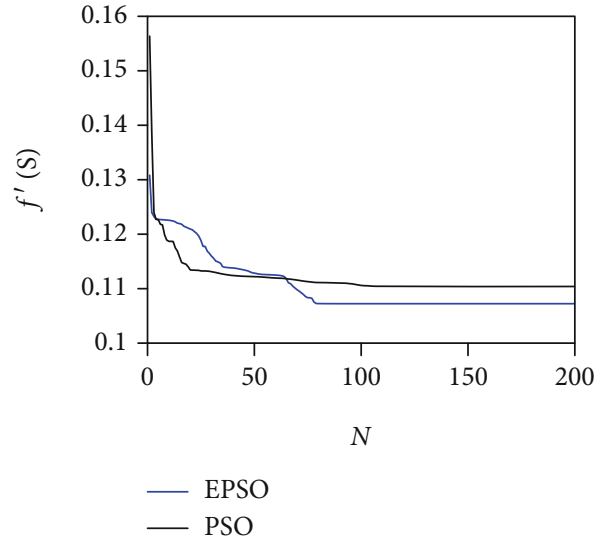

Figure 6: Calculation results of $f^{\prime}(\mathbf{S})$ under different algorithms.

As can be seen in Figure 6, the EPSO results stabilize at the 86th calculation; i.e., the nodal injection power combination where the power grid is in the critical state of cascading trips is found. The minimum $f^{\prime}(\mathbf{S})$ value is 0.5446 , and the corresponding minimum $f(\mathbf{S})$ value is 0.4799 . While the PSO results stabilize by the 66th calculation, the minimum $f^{\prime}(\mathbf{S})$ is 0.1104 and the corresponding $f(\mathbf{S})$ is 0.0883 . The comparison results show that EPSO has some advantages in calculating the minimum $f^{\prime}(\mathbf{S})$, so EPSO is more suitable to be chosen. EPSO is used to obtain the nodal injection power in the $\mathbf{S}$ state corresponding to the minimum $f^{\prime}(\mathbf{S})$, as shown in Table 3.

Table 3 shows the node injected power for the IEEE39 node system listed by the PQ node and PV node, respectively. For the PQ node, Table 1 lists the active and reactive power at its nodes, with active power on the left and reactive power on the right. For the PV node, Table 3 lists only the active power injected at the nodes. In Table 3, the unit of active power is MW, and the unit of reactive power is MVAR. If the power data in the table is positive, it means that the actual flow direction of this power is the outflow from the node. If it is negative, it means that the actual flow direction of this power is injected into the power grid from the node.

To verify the results obtained by EPSO in Figure 6, this paper uses the current and voltage collected by WSN to calculate the nodal injection power data according to Equation (2). Based on this data, by randomly modifying the power on each node, several nodal injection power states for comparison are obtained. Then, the $f^{\prime}(\mathbf{S})$ of the power grid in these states are calculated, respectively, and they are compared with the minimum $f^{\prime}(\mathbf{S})$ obtained by EPSO in Figure 6. The corresponding calculation results are shown in Figure 7. The ordinate of Figure 7 is the $f$ '(S) in the form of per-unit value, and the abscissa $N_{\mathrm{s}}$ is the serial number of the nodal injection power state, where the first injection power state is the corresponding one in Table 3.

In this paper, the injection power state of each node used for comparison in Figure 7 is formed as follows: according to the injection power state corresponding to the minimum $f^{\prime}$ 
TABle 3: The nodal injection power corresponding to the $S$ state obtained by EPSO.

\begin{tabular}{|c|c|c|c|}
\hline & Node number & $P(\mathrm{MW})$ & Q (MVAR) \\
\hline \multirow{17}{*}{ Load nodes (PQ) } & 3 & -322 & -2.405 \\
\hline & 4 & -500 & -184 \\
\hline & 7 & -233.8 & -84.001 \\
\hline & 8 & -522 & -176 \\
\hline & 12 & -8.512 & -88.002 \\
\hline & 15 & -330 & -153 \\
\hline & 16 & -329 & -32.293 \\
\hline & 18 & -158 & -29.992 \\
\hline & 20 & -680 & -103 \\
\hline & 21 & -274 & -115 \\
\hline & 23 & -247.5 & -84.601 \\
\hline & 24 & -308.6 & 92.198 \\
\hline & 25 & -224 & -47.197 \\
\hline & 26 & -139 & -16.995 \\
\hline & 27 & -281 & -75.499 \\
\hline & 28 & -206 & -27.591 \\
\hline & 29 & -283.5 & -26.891 \\
\hline \multirow{9}{*}{ Power nodes (PV) } & 30 & 250 & \\
\hline & 32 & 650 & \\
\hline & 33 & 632 & \\
\hline & 34 & 508 & \\
\hline & 35 & 650 & \\
\hline & 36 & 560 & \\
\hline & 37 & 540 & \\
\hline & 38 & 830 & \\
\hline & 39 & 1000 & \\
\hline
\end{tabular}

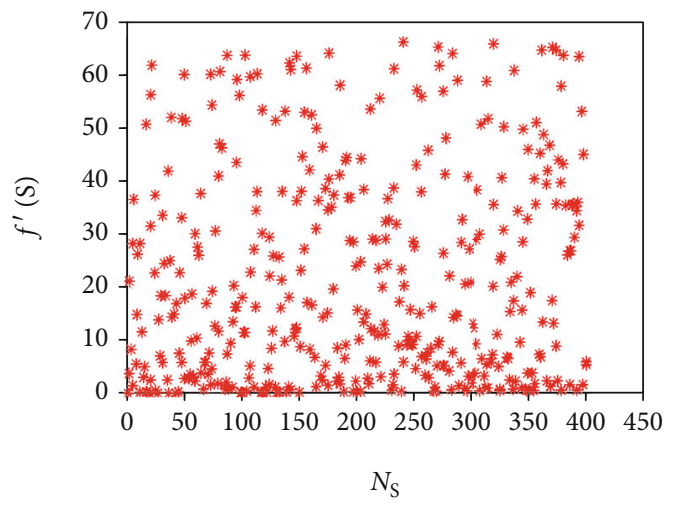

Figure 7: The $f^{\prime}(\mathbf{S})$ corresponding to the injection power state of each node during verification.

(S) in Figure 6, the power of each node $i$ was modified according to the following equation:

$$
S_{i}^{\prime}=S_{i}+0.1 \times q_{1} \times q_{2} .
$$

Here, $q_{1}$ is a random number uniformly distributed over

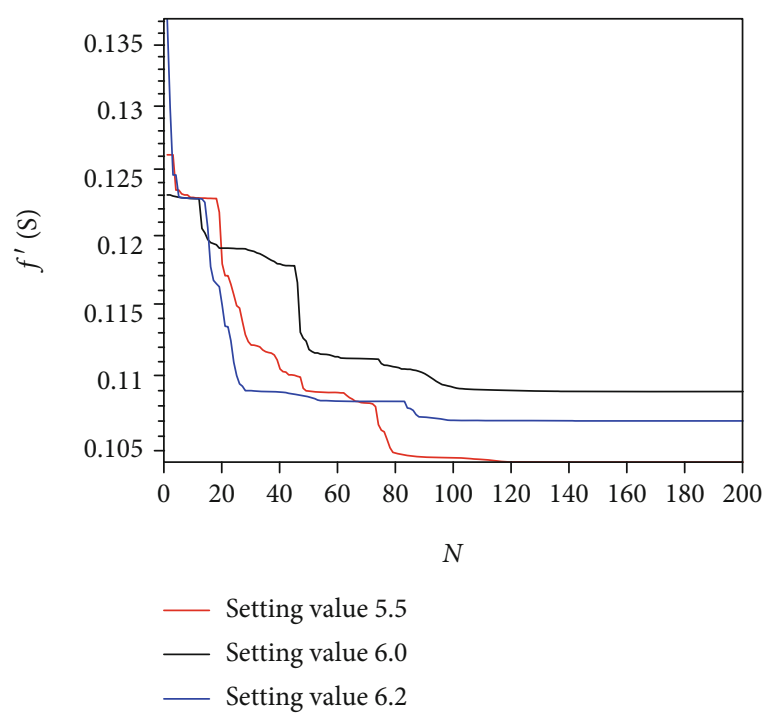

FIgURE 8: Calculation results of $f^{\prime}(\mathbf{S})$ with different protection setting values.

the $(0,1)$ interval, while $q_{2}$ is a number obtained by $q_{1}$. If $q_{1}$ $>0.5$, then $q_{2}$ is taken as 1 , and if $q_{1} \leq 0.5$, then $q_{2}$ is taken as -1 .

After comparison, it can be seen from Figure 7 that the minimum $f^{\prime}(\mathbf{S})$ obtained by EPSO in Figure 6 is also minimum in Figure 7, thus verifying the results in Table 3. The results obtained in this paper are similar to those in Figure 7 for several validations along the above lines, which are not drawn here due to the limitation of space. In conclusion, it can be seen from these comparisons that the minimum $f^{\prime}(\mathbf{S})$ obtained by EPSO in Figure 6 is acceptable.

To further verify the method in this paper, EPSO is used to analyze a large number of examples for different relay setting values. The calculation of $f^{\prime}(\mathbf{S})$ is similar to that in Figure 6. For the convenience of illustration, Figure 8 shows the calculation of three different protection settings. The meaning of ordinate and abscissa in Figure 8 is the same as that in Figure 6, and the protection setting values corresponding to each curve are shown in Figure 8.

As seen in Figure 8, the trend of $f^{\prime}(\mathbf{S})$ changes during the iteration process is similar to that of Figure 6 , and its value finally converges after a series of irregular decreases from the beginning of the iteration. In this paper, in addition to quantitative analysis for different protection setting values, for the initial fault, in addition to branch $L_{5,8}$ as the initial fault branch for calculation, for other branch roads composed of lines or transformers in the power grid, different branch roads are selected as the initial fault, and for different protection setting values for quantitative analysis and calculation, the calculation results are similar to Figure 8, while after a comparative analysis similar to Figures 6 and 7, the results are satisfactory, which shows that the model and solution ideas given in this paper are reasonable.

In the example, after calculating $f(\mathbf{S})$ and $f^{\prime}(\mathbf{S})$, a safe region $C_{1}$ similar to the one shown in Figure 4 is obtained. For a given initial failure, assuming several operational states 
are in region $C_{1}$, the current of each branch of the power grid is calculated by using the power flow calculation. According to Equation (5), it is determined that the power grid will not be cascading trips. Since the WSN can quickly and effectively collect the voltage and current of the power grid, the collected voltage and current are consistent with the calculation results of power flow. Therefore, it shows that WSN can be used to verify the previous calculation.

In short, through the above example, we can find that the corresponding $f^{\prime}(\mathbf{S})$ and $\mathbf{S}$ can be obtained by using the method in this paper. The $f^{\prime}(\mathbf{S})$ can help the operators to observe the distance between the current power grid and the critical state of the cascading trips, and $\mathbf{S}$ can alert the operators to the specific location of the closest critical state, thus providing a basis for the operator to avoid the power grid entering the critical position.

\section{Conclusion}

This paper studies the nodal injection power data of the power system based on WSN calculations and transmits these data to the monitoring center, and the monitors use the nodal injection power data to evaluate the security of the current power grid, and the paper gives the relevant analysis models and evaluation methods, and the main conclusions are as follows:

(1) As an ideal choice to deal with the new challenges of power grid parameter monitoring technology, WSN provides a more flexible and perfect solution for power grid parameter monitoring and can realize the centralized management of multitype power grid parameter monitoring. Using WSN, the power injection data of power grid nodes can be given quickly, accurately, and comprehensively. With the corresponding security level analysis algorithm, it can quickly and accurately evaluate the online security of the power system

(2) Since the cascading trips of the power grid are closely related to the action behavior of the relay protection, the mathematical expression of the cascading trips of the power grid can be given by the distillation of the action equation of the protection

(3) The SLP method mainly uses the nodal injection power of the grid to obtain the current operating state of the grid and the security evaluation after the migration of the operating state, which is simple and practical

(4) This paper presents a model to analyze the security level of the power grid for cascading trips, which can be used not only to calculate the security level of the power grid for cascading trips but also to calculate the initial critical operation state closest to the current operation state of the power grid, which provides a strong basis for avoiding the power grid entering the critical state and thus avoiding cascading trips
In a word, the method proposed in this paper can provide a reference for further research on cascading fault of the power grid and provide theoretical and technical support for the actual operation of the power grid.

\section{Data Availability}

The data used to support the findings of this study are available from the corresponding author upon request.

\section{Conflicts of Interest}

The authors declare that they have no conflicts of interest.

\section{Acknowledgments}

This research is supported by the Scientific Research Development Foundation of the Fujian University of Technology under the grant GY-Z17149 and the Scientific and Technological Research Project of Fuzhou under the grant GYZ18058.

\section{References}

[1] M. Zhou, Q. Liang, H. Wu, W. Meng, and K. Xu, "Wireless sensor networks for smart communications," Wireless Communications and Mobile Computing, vol. 2018, Article ID 4727385, 2 pages, 2018.

[2] D. Zhi-gang and S. Teng-fei, "Development status and application prospect of electronic current transformer," Instrumentation Technology, vol. 2019, no. 5, pp. 37-44, 2019.

[3] B.-S. Kim, H. S. Park, K. H. Kim, D. Godfrey, and K.-I. Kim, “A survey on real-time communications in wireless sensor networks," Wireless Communications and Mobile Computing, vol. 2017, Article ID 1864847, 14 pages, 2017.

[4] V. C. Gungor, B. Lu, and G. P. Hancke, "Opportunities and challenges of wireless sensor networks in smart grid," IEEE Transactions on Industrial Electronics, vol. 57, no. 10, pp. 3557-3564, 2010.

[5] F. Fan, Q. Ji, G. Wu, M. Wang, X. Ye, and Q. Mei, "Dynamic barrier coverage in a wireless sensor network for smart grids," Sensors, vol. 19, no. 1, pp. 3557-3564, 2019.

[6] Z.-j. Bao, W.-j. Yan, and G. Wu, "Control of cascading failures in coupled map lattices based on adaptive predictive pinning control," Journal of Zhejiang University-Science C(Computers \& Electronics), vol. 12, no. 10, article 1258, pp. 828-835, 2011.

[7] J. Xu, X. Bai, and B. Huang, "Research on wide area cooperative precontrol system for cascading trips," Power System Technology, vol. 37, no. 1, pp. 131-136, 2013.

[8] M. Tian, X. Wang, Z. Dong et al., "Cascading failures of interdependent modular scale-free networks with different coupling preferences," Epl, vol. 111, no. 1, article 18007, 2015.

[9] Y. F. Wang, K. L. Gao, T. Zhao, and J. Qiu, "Assessing the harmfulness of cascading trips across space in electric cyberphysical system based on improved attack graph," Proceedings of the CSEE, vol. 36, no. 6, pp. 1490-1499, 2016.

[10] A. Hilmani, A. Maizate, and L. Hassouni, "Automated realtime intelligent traffic control system for smart cities using wireless sensor networks," Wireless Communications and 
Mobile Computing, vol. 2020, Article ID 8841893, 28 pages, 2020.

[11] B. Wang, J. Sen, and Z. Shaomin, "Research on WSN topology and protocol for transmission lines monitoring," Information Systems and Signal Processing Journal, vol. 4, no. 1, 2019.

[12] B. Fateh, M. Govindarasu, and V. Ajjarapu, "Wireless network design for transmission line monitoring in smart grid," IEEE Transactions on Smart Grid, vol. 4, no. 2, pp. 1076-1086, 2013.

[13] L. I. Miao, C. Xiaobo, J. Xinchun et al., "Monitoring for overhead transmission lines based on WSN in smart grid," Shaanxi Electric Power, vol. 44, no. 10, pp. 1-5, 2016.

[14] X. Xue and J. Lu, "A compact brain storm algorithm for matching ontologies," IEEE Access, vol. 8, no. 8, pp. 4389843907, 2020.

[15] X. Xue and Y. Wang, "Optimizing ontology alignments through a memetic algorithm using both MatchFmeasure and unanimous improvement ratio," Artificial Intelligence, vol. 223, no. 223, pp. 65-81, 2015.

[16] X. Xue and Y. Wang, "Using memetic algorithm for instance coreference resolution," IEEE Transactions on Knowledge and Data Engineering, vol. 28, no. 2, pp. 580-591, 2016.

[17] X. Xue and J. Chen, "Optimizing sensor ontology alignment through compact co-firefly algorithm," Sensors, vol. 20, no. 7, pp. 2056-2115, 2020.

[18] X. Xue, "A compact firefly algorithm for matching biomedical ontologies," Knowledge and Information Systems, vol. 62, article 1443, no. 7, pp. 2855-2871, 2020.

[19] Y. Zhiqiang, H. Zhijian, and J. Chuanwen, "Economic dispatch and optimal power flow based on chaotic optimization," Proceedings. International Conference on Power System Technology, vol. 2002, no. 4, pp. 2313-2317, 2002.

[20] G. Shanghong and P. Tinglong, "Photovoltaic electricity generation power prediction based on similar day and LS-SVM with EPSO," Transducer and Microsystem Technologies, vol. 38, no. 3, pp. 40-46, 2019.

[21] S. Lian, S. Meng, and Y. Wang, "An objective penalty functionbased method for inequality constrained minimization problem," Mathematical Problems in Engineering, vol. 2018, Article ID 7484256, 7 pages, 2018. 